\title{
Algae to the Rescue: Infusing engineering into High School Biology
}

\section{Katheryn Kennedy, CIESE, Stevens Institute of Technology}

Kathy Kennedy is a Program Manager for CIESE at Stevens Institute of Technology. Kathy is an experienced college, high school, and middle school science teacher and has led numerous K-12 teacher professional development programs in engineering and STEM education. Kathy holds a degree in biology from Siena College, a Masters in Biomedical Science from Baylor University, and is currently enrolled in a doctoral program at Walden University. 


\section{WORKSHOP PROPOSAL FORM}

2015 Annual ASEE K-12 Workshop on Engineering Education

"Authentic Engineering: Representing \& Emphasizing the E in STEM"

Presented by Dassault Systems

Saturday, June 13, 2015

8:00 A.M. - 5:00 P.M.

Sheraton Seattle | Seattle |WA

Please complete this form, save it as a PDF file only and upload it through the ASEE Paper Management system as shown in the K12 Workshop Presenter's Kit.

All notifications will be by email from the ASEE Paper Management system.

NOTE: To ensure that emails are not obstructed by spam blockers, please make sure to WHITELIST the email addresses: monolith@asee.org and conferences@ asee.org and s.harrington-hurd@ asee.org.

Direct questions to Stephanie Harrington-Hurd, ASEE K-12 Activities Manager, at s.harringtonhurd@asee.org. Additional workshop details are available at: http://www.asee.org/K12Workshop. Thank you!

Deadline

Friday, January 23, 2015 by 5:00PM EST

Presenters will be notified of acceptance status by March 14. Late submissions will not be accepted. Advanced Workshop Registration will open December 6, 2013.

\section{SUBMISSION INFORMATION}

Provide the first and last name of each presenter, including affiliations. If there is more than one presenter, designate one person as the organizer and provide only that person's contact information. The organizer is responsible for communicating to co-presenters.

Number of Presenters: 1

Presenter Name(s):

1) Last Kennedy First Katheryn Affiliation Stevens Institute of Technology, CIESE

Contact Person's Name: Katheryn Kennedy

Contact Person’s Email: Katheryn.Kennedy@ stevens.edu

Contact Person's Phone: 201-216-5443

Contact Person's Alternate Phone: 


\section{WORKSHOP PROPOSAL FORM}

2015 Annual ASEE K-12 Workshop on Engineering Education

"Authentic Engineering: Representing \& Emphasizing the E in STEM"

Presented by Dassault Systems

Saturday, June 13, 2015

8:00 A.M. - 5:00 P.M.

Sheraton Seattle | Seattle |WA

Please provide a one-paragraph bio for each presenter (in the order listed above). The bio should not exceed 70 words and should be written as you would want it to appear on the ASEE website and program materials.

1) Kathy Kennedy is a Program Manager for CIESE at Stevens Institute of Technology. Kathy is an experienced college, high school, and middle school science teacher and has led numerous K-12 teacher professional development programs in engineering and STEM education. Kathy holds a degree in biology from Siena College, a Masters in Biomedical Science from Baylor University, and is currently enrolled in a doctoral program at Walden University.

2)

3)

\section{WORKSHOP INFORMATION}

\section{Proposed Title:}

Algae to the Rescue: Infusing engineering into High School Biology

Abstract: Please provide a concise description that includes the workshop's learning objectives (maximum 750 characters). The abstract is used on the ASEE website, program materials, and otherK-12 Workshop promotional activities.

Carbon Imbalance: Algae to the Rescue is an NSF-funded curriculum module in which biology students are introduced to the engineering design process through a design challenge. As they progress through the module, students develop connections among plants, atmospheric carbon dioxide levels, combustion, and energy. In this workshop, participants will engage in an engineering redesign activity in which they optimize a device that will feed the combustion gases from a burning candle into the environment of an aquatic plant, Elodea. This device or process is a subsystem that will eventually be used or adapted for their prototype algae farm.

Workshop Description. Please provide a detailed description of the proposed workshop that, at minimum, explicitly addresses the following (maximum 4,000 characters):

a. Learning objectives

b. Hands-on activities and interactive exercises 


\section{WORKSHOP PROPOSAL FORM}

2015 Annual ASEE K-12 Workshop on Engineering Education

"Authentic Engineering: Representing \& Emphasizing the E in STEM"

Presented by Dassault Systems

Saturday, June 13, 2015

8:00 A.M. - 5:00 P.M.

Sheraton Seattle | Seattle | WA

c. Materials that participants can take with them

d. Practical application for teachers and outreach staff

In this session participants will be presented with an overview of the module to provide context and discuss the biology concepts supported through the design challenge. Carbon imbalance is related to the topics of photosynthesis, respiration and the cycling of matter within the carbon cycle and placed within the context of human impact. An engineering design challenge to address this issue centers on designing an algae farm to sequester CO2. Participants in the workshop will be engaged in an engineering redesign activity that will improve one of the subsystems of the algae farm.

Participants will be able to:

1. Apply the engineering design process to a narrowly focused problem.

2. Make their thought processes apparent through discussion and design packets

3. Develop connections among plants, atmospheric carbon dioxide levels, combustion, and energy through discussion

Hands-on activities include:

1. Design/redesign the carbon capture unit delivery subsystem that serves as part of the algae farm. Participants working in groups of 4 will redesign and optimize a device that will feed the combustion gases from a burning candle into the environment of an aquatic plant. This device will eventually be used or adapted for their algae farms. Common devices resemble smokestacks and are constructed from recycled materials such as soup cans, coffee cans, glass funnels, tubing, clay, tape, and a candle. Groups must focus on the construction of the device and address issues of keeping the candle burning and collecting the $\mathrm{CO} 2$ that is given off from the candle. Groups must also consider how they will collect the $\mathrm{CO} 2$ gas and transfer it to the algae farm.

2. Groups will test the effectiveness of their delivery system by using bromothymol blue as an indicator to reveal the presence of $\mathrm{CO} 2$ coming from a collection/transfer tube.

3. Participants will document the process through the use of a design packet that will facilitate writing and sketching of the device as well as construction or design changes made to optimize effectiveness.

4.

Materials for participants:

1. Handout to facilitate the redesign activity.

2. Design packet to document iterative design or construction changes made to optimize the product in both writing and with drawings.

3. Carbon capture prototype (1 per team)

4. Access to freely available NSF funded module Carbon Imbalance: Algae to the Rescue 


\title{
WORKSHOP PROPOSAL FORM
}

\author{
2015 Annual ASEE K-12 Workshop on Engineering Education \\ "Authentic Engineering: Representing \& Emphasizing the E in STEM" \\ Presented by Dassault Systems \\ Saturday, June 13, 2015 \\ 8:00 A.M. - 5:00 P.M. \\ Sheraton Seattle | Seattle |WA
}

Practical applications:

One of the reasons that global environmental issues are so difficult to solve is the complexity of environmental problems. While their complexity also contributes to the challenge of incorporating these issues in the classroom, it allows for rich educational experiences connecting topics within biology and connecting biology with chemistry and engineering. This module engages students in hands-on lab activities, a virtual lab, and an engineering design activity. The initial activities are very structured, much like traditional lab investigations, while the later activities are more open. The engineering design activities are related to atmospheric carbon dioxide and require students to apply and synthesize concepts related to photosynthesis and the cycling of carbon in living and nonliving components of the biosphere as well as gain a basic understanding of engineering concepts and introductory level of related skills. It addresses common misconceptions regarding gas exchange among organisms and issues surrounding global climate change. The module is intended to be implemented in the classroom after teaching photosynthesis and respiration or in conjunction with an ecology unit. The social perspectives, technology, and engineering standards and objectives are addressed as students engage in an extended design activity.

Authentic Engineering Connection. Identify and describe how you will explicitly address the ways in which your lesson or activity is representative of the processes, habits of mind and practices used by engineers, or is demonstrative of work in specific engineering fields. ${ }^{i}$ At least one of those must be within the first four listed, below; i.e., do not only check "other". Check all that apply:

$\mathrm{X}$ Use of an engineering design process that has at least one iteration/improvement $\square$ Attention to specific engineering habits of mind

$\mathrm{X}$ Attention to engineering practices (as described in the NGSS/Framework and as practiced by engineers)

$\square$ Attention to specific engineering careers or fields related to the lesson/activity Other (please describe below)

Provide a description of how you will explicitly address these aspects of authentic engineering in your workshop (maximum 2,000 characters):

In our use of the module with students thus far, the design/redesign of the carbon capture requires several iterations to optimize the ability to gather $\mathrm{CO} 2$ waste from a burning candle. The candle models fossil fuel burning and represents the human contribution to climate change. The framing of the design challenge within the module engages students to consider human sources of $\mathrm{CO} 2$ and optimize a possible solution, considering requirements and constraints presented in a design brief. 


\section{WORKSHOP PROPOSAL FORM}

2015 Annual ASEE K-12 Workshop on Engineering Education

"Authentic Engineering: Representing \& Emphasizing the E in STEM"

Presented by Dassault Systems

Saturday, June 13, 2015

8:00 A.M. - 5:00 P.M.

Sheraton Seattle | Seattle |WA

Highlighting the data collection of information gained in the testing phases and using the data to inform design choices is explicit in the student packet which also captures brainstorming, research, and sketches of redesign.

The NGSS engineering standards addressed by this module include:

HS-ETS1-1 Analyze a major global challenge to specify qualitative and quantitative criteria and constraints for solutions that account for societal needs and wants.

HS-ETS1-2 Design a solution to a complex real-world problem by breaking it down into smaller, more manageable problems that can be solved through engineering.

Diversity. This year is the American Society for Engineering Education's "Year of Action on Diversity." It is essential that we have a diverse engineering workforce to solve diverse problems. To do that and to have an engineering-literate public, it is essential that we reach every preK-12 student with high-quality engineering education, drawing on issues of access and equity in the classroom and in the curriculum. Reviewers would like to know how your proposed workshop will address diversity.

Provide a description of how you will explicitly address diversity - e.g., diversity with respect to gender/sex, ethnicity or race, special education inclusion, socio-economic status, or LGBT status - in your workshop (maximum 2,000 characters):

The hands-on activity will address engagement of all participants within the workshop. Providing a design challenge with a context to help others will increase engagement for all students and particularly for females within this science course.

Special education students tend to perform well in hands-on challenges and this task provides opportunities for success.

Are there any online components to the proposal or presentation? (Note that these online components may only be available to presenters or those who have their wireless subscriptions, since wireless may not be available during the workshop sessions.)

$$
\begin{aligned}
& \text { X No } \\
& \square \text { Yes }
\end{aligned}
$$

Please describe:

Grade Level Target Audience (check all that apply): 


\section{WORKSHOP PROPOSAL FORM}

2015 Annual ASEE K-12 Workshop on Engineering Education

"Authentic Engineering: Representing \& Emphasizing the E in STEM"

Presented by Dassault Systems

Saturday, June 13, 2015

8:00 A.M. - 5:00 P.M.

Sheraton Seattle | Seattle | WA

Primary (EC-2)

Elementary (3-5)

Middle School (6-8)

X High School (9-12)

Maximum Number of Participants:

25

If this number is greater than 25 , please describe how your workshop will equally engage all participants.

All Seating is Classroom (tables and chairs).

Audio Visual Equipment Requests:

Note: An LCD projector, screen and podium with attached microphone are provided. Requests for additional equipment or resources (e.g., internet connection or laptops) will incur extra charges. If you do not have additional requests, please indicate with "Not applicable."

Not Applicable

Reminder:

Presenters must register and pay the registration fee to support their workshop attendance and audio/video costs.

Thank you for completing this proposal form!

Please review this document prior to submitting it to ensure that all items are complete.

Date Received:

Received By:

Proposal ID Number: 